\title{
Sobre la formación de recursos humanos para la ciencia
}

\author{
Coppo, J.A.
}

Cátedra de Fisiología, Facultad de Ciencias Veterinarias, Universidad Nacional del Nordeste (UNNE),

Sargento Cabral 2139, Corrientes (3400), Argentina. E-mail: jcoppo@vet.unne.edu.ar.

\begin{abstract}
Resumen
Coppo, J.A.: Sobre la formación de recursos humanos para la ciencia. Rev. vet. 24: 1, 66-75, 2013. La formación de recursos humanos para investigación científica irrumpió hace algunas décadas en las universidades argentinas como actividad ineludible para la continuidad de la carrera de docencia e investigación. El objetivo de esta revisión historiográfica fue rastrear los inicios de la relación científico-discípulo, así como los resultados emanados de tal asociación. A partir del Siglo XVII sobresalen por sus descubrimientos las figuras de discípulos como Ham, van Swieten, Auenbrugger, Schlemm, Langerhans, Ranvier, Sandström, Bernstein, Guérin, Allen, Price, Bertram, Huxley, Varela y Best, entre otros. Se describen los logros iniciales obtenidos por estos jóvenes, a veces emulando a sus tutores, así como su ulterior consolidación como científicos de renombre. Se concluye que la formación de recursos humanos existió a partir de los inicios de la "ciencia normal" y que su efecto multiplicador es altamente provechoso para el incremento del conocimiento. Se resalta el carácter azaroso de la admisión del discípulo, así como el imperfecto sistema de valoración del tutor, que privilegia la cantidad y no la calidad de los discípulos.
\end{abstract}

Palabras clave: recursos humanos, ciencia, historia, beneficios, inconvenientes.

\begin{abstract}
Coppo, J.A.: About the formation of human resources in science. Rev. vet. 24: 1, 66-75, 2013. Formation of human resources for scientific investigation started some decades ago in Argentinean universities as an unavoidable activity for the continuity of teaching and investigation career. The objective of this historical revision was to know the beginnings of the scientific-disciple relationship, as well as the results consequent to such association. Starting from the XVII Century, disciples such as Ham, van Swieten, Auenbrugger, Schlemm, Langerhans, Ranvier, Sandström, Bernstein, Guérin, Allen, Price, Bertram, Huxley, Varela and Best, among others, stand out for their discoveries. Initial achievements of these young men, sometimes resembling their tutors, as well as their ulterior consolidation as a distinguished scientific, are described. It is concluded that the formation of human resources started from the beginnings of the "normal science" and that its multiplying effect is highly beneficial on behalf of knowledge. The risky character of the disciple's admission, as well as the imperfect system of tutor's valuation - privileging quantity rather than quality of the disciplines- are emphasized.
\end{abstract}

Key words: human resources, science, history, benefits, inconveniences.

\section{INTRODUCCIÓN}

Un discípulo a quien jamás se le pide nada que no pueda hacer, nunca hará todo lo que puede.

John Stuart Mill

Originariamente, la búsqueda de conocimientos científicos fue unipersonal. Hasta finales del Siglo XIX la gran mayoría de los descubrimientos fue realizado por un investigador "solitario". Prueba de ello

Recibido: 20 diciembre 2012 / Aceptado: 27 febrero 2013 son los hallazgos de Copérnico, Galileo, Servet, Harvey, Malpighi, Newton, Linné, Lavoisier, Spallanzani, Galvani, Volta, Jenner, Purkinje, Schwann, Darwin, Mendel, Pasteur, Koch, Golgi, Pavlov, Bernard, Cannon, Lorenz y muchos otros. Desde finales del Siglo XIX en adelante, la interacción entre científicos se tradujo en descubrimientos realizados a dúo, como los de Chauveau y Marey (presión arterial), Hering y Breuer (ventilación pulmonar), Hertwig y Fol (fecundación del óvulo), Salmon y Smith (bacteriología), Minkowski y von Mering (función pancreática), Bayliss y Starling (secretina, primera hormona), Keith y Flack (marca- 
paso cardíaco), Murlin y Kimball (hormona glucagon), Aschheim y Zondek (HCG, test de preñez), Landsteiner y Wiener (factor Rh), Milstein y Köhler (anticuerpos monoclonales), entre otros. Hacia mediados del Siglo $\mathrm{XX}$ proliferaron equipos integrados por numerosos investigadores, muchos de ellos con extraordinaria producción científica, como el liderado en Argentina por el Premio Nobel Bernardo Houssay (Fisiología, 1947), grupo que incluyó personalidades de la talla de Leloir, Braun-Menéndez, Hug, Lewis, Orías, Foglia, Taquini, Muñoz, Fasciolo y otros ${ }^{6}$.

Muchas veces la pluralidad implicó que los investigadores admitieran la participación de algún principiante en su proyecto científico. A veces se trataba de un recientemente egresado universitario y en otros casos de estudiantes avanzados ("ayudantes alumnos", "asistentes", "pasantes"), los cuales al cabo de un tiempo se transformaban en discípulos ("becarios", "tesistas"), figurando con su mentor en las publicaciones científicas. Tal formación de recursos humanos generó un efecto multiplicador que redundó en una extraordinaria prolificidad, a punto tal que en muchos casos los discípulos culminaron desplegando una producción científica que emuló a la de sus tutores y produjo un extraordinario avance del conocimiento ${ }^{5}$.

En términos kuhneanos toda ciencia tuvo una etapa de pre-ciencia (ausencia de paradigma), en la cual distintas "escuelas" discutieron distintas posturas, hasta alcanzar un paradigma (marco, modelo, perspectiva), dentro del cual se desarrollaría la ciencia normal, que debería perdurar hasta que una revolución científica cambiara el paradigma. Un discípulo o ayudante alumno aprende y reproduce la erudición de su maestro, adoptando la misma manera de practicar la ciencia normal. Ambos trabajan dentro del marco del paradigma aceptado sin discutirlo ni defenderlo, dedicándose a resolver problemas y generar nuevos conocimientos ${ }^{16}$.

Los científicos son los productores y validadores del conocimiento, son individuos de formación similar, resolvedores de problemas atinentes a una especialidad. Según Kuhn, poseen una educación similar y una bibliografía técnica común, estrecha comunicación entre sí y juicios profesionales homogéneos, casi unánimes. Obtienen lecciones equivalentes y están convencidos de su responsabilidad en la investigación de un conjunto de objetos comunes ${ }^{16}$. He aquí cuán importante es que un científico forme (modele, construya) discípulos, incluso reclutándolos a partir de su etapa de estudiante. Hace algún tiempo, la tan mentada "formación de recursos humanos" apareció como un requisito "novedoso" en universidades e institutos de investigación, a manera de fulgurante estrella que iluminaría el sendero conducente al futuro e inminente desarrollo, amén de constituir una cuasi obligación para el sostenimiento y avance de la carrera universitaria y científica. Pero ¿era realmente tan innovadora?

El objetivo de esta breve revisión fue efectuar, a través de técnicas heurísticas, una indagación historiográfica que documente cuán reciente e innovadora es la tarea de formación de recursos humanos para la investigación en "ciencias fácticas", especialmente en fisiología por ser la disciplina del autor de este escrito.

\section{DESARROLLO}

A partir de una gran cantidad de casos estudiados, se seleccionaron algunos que resultan paradigmáticos por la trascendencia de los personajes involucrados y por la magnitud de los descubrimientos efectuados.

Johan Ham, estudiante de medicina holandés nacido presumiblemente en 1654, era el ayudante del naturalista Anton van Leeuwenhoek, cuyo pasatiempo de pulidor de cristales lo llevó a fabricar el primer microscopio. El objeto a examinar, montado sobre la cabeza de un alfiler, era ampliado unas 300 veces. En 1668 el maestro confirmó el descubrimiento de la red de capilares efectuado por Malpighi, demostrando la circulación de la sangre por los vasos de la oreja de un conejo y por la membrana interdigital de la pata de una rana. También describió la estriación transversal de las fibras musculares, la naturaleza reticular del miocardio y la estructura fibrilar del cristalino. En agua estancada observó "animáculos", lo cual constituiría la primera mención de los actuales protozoarios. Leeuwenhoek se enfrentó a la teoría de la generación espontánea al afirmar que los animales diminutos no surgían espontáneamente, sino que se desarrollaban a partir de "huevecillos". En 1675, con el primitivo microscopio disponible, su ayudante Ham descubrió los espermatozoides, a los cuales consideró como "animáculos seminales", una especie de gusanos móviles, de finas y largas colas ${ }^{10}$ (Figura 1).

Maestro y alumno profundizaron el tema concluyendo que los espermatozoides eran pequeños corpúsculos vivos, normales en el semen de cualquier animal, en contradicción a las teorías imperantes sostenidas por Harvey y Fabricius, derivadas de las ideas de Aristóteles y Galeno. En 1678 se realizó la primera descripción precisa de los "glóbulos rojos" 28 . Leeuwenhoek y Ham efectuaron un descubrimiento muy importante, al reconocer la verdadera naturaleza de los microorganismos. Asumiendo que la vida y la motilidad son recíprocas, concluyeron que los objetos móviles vistos a través del

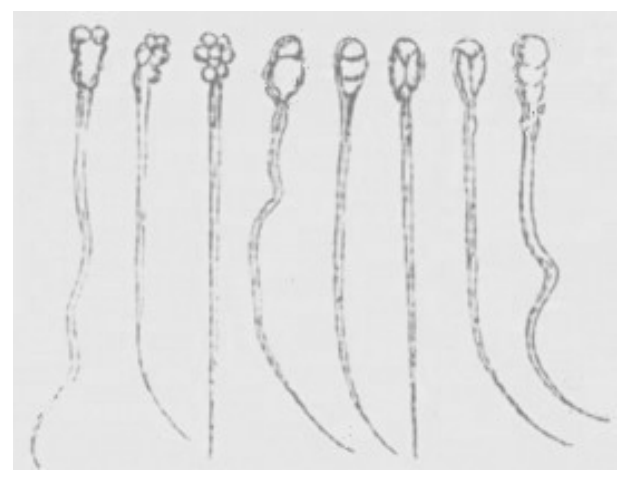

Figura 1. Primeros espermatozoides observados. 
microscopio eran pequeños animales, relato que causó gran conmoción al ser expuesto en la Royal Society de Londres. Así fueron descriptos muchos microorganismos, incluso bacterias, protozoarios y rotíferos. A partir del hallazgo de su ayudante alumno, en el curso de cuarenta años Leeuwenhoek describió los espermatozoides de artrópodos, moluscos, peces, anfibios, aves y mamíferos ${ }^{10}$.

Gerhard van Swieten (1700-1772) fue discípulo y asistente de Herman Boerhaave. Este último, médico y botánico holandés, profesor de la Universidad de Leiden, tuvo una gran influencia en el avance de la química, la botánica y la medicina. Van Swieten asistió a Boerhaave en sus experimentos químicos, en una época en que predominaban las creencias de la alquimia, desterrando algunos de sus postulados. Demostraron que la "materia ígnea" no tiene peso, y que una masa de hierro pesa lo mismo a temperatura ambiente que cuando está al rojo. En 1729 logran aislar en orina la sal netivis urinae, cuya naturaleza química (urea) fue dilucidada 50 años más tarde. En 1731 Boerhaave publicó su libro Elementa Chemiae, quizás el más importante de los primitivos textos de química, ya que recogió todos los conocimientos que se tenían entonces acerca de tal disciplina. En medicina estudió la fiebre y la inflamación; también elaboró una teoría sobre la circulación de la sangre y la conformación de los vasos sanguíneos, incluyendo las patologías que se producían en ellos. Fue el primero en describir la ruptura de esófago con salida del contenido gástrico hacia el mediastino, entidad conocida hoy como Síndrome de Boerhaave. A su discípulo van Swieten se le atribuye el mérito de introducir la técnica de la percusión como maniobra semiológica (percutía el abdomen para delimitar la magnitud de la ascitis) ${ }^{19}$. Van Swieten también asumió la ímproba tarea de compendiar y comentar los libros de su maestro, especialmente los cinco volúmenes de "Aforismos" (Figura 2).

Afincado en Viena, van Swieten se ocupó de ampliar los conocimientos de anatomía, fisiología y pa-

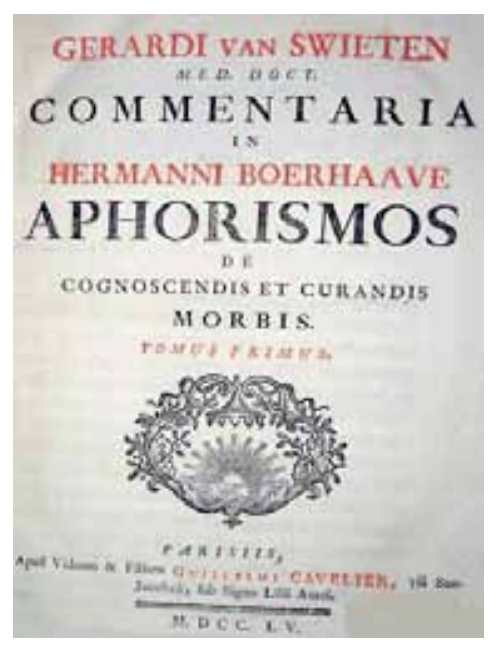

Figura 2. Facsímil de "Aforismos" (1755).

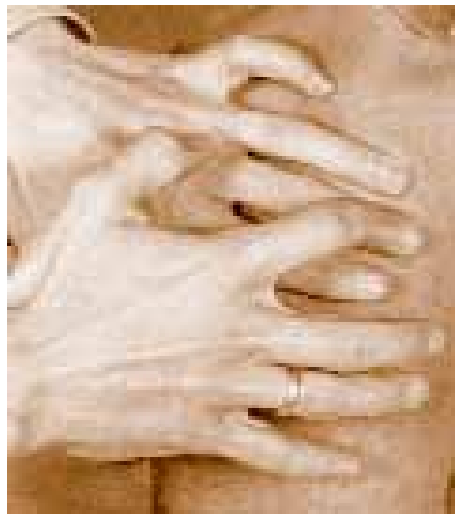

Figura 3. Técnica de percusión.

tología, así como de mejorar el tratamiento de las enfermedades venéreas, creando un elixir que trataba la sífilis. Con él se cerró la época de enseñanza libresca y se inauguró otra presidida por la nova scientia (medicina basada en la observación y experimentación) que él aprendió de su maestro Boerhaave. Van Swieten, rígido en su metodología, era conocido también por hablar varias lenguas, ser muy generoso con los pobres y destinar parte de sus ganancias para ayudar a los estudiantes con talento ${ }^{7}$.

Leopold Auenbrugger (1722-1809), estudiante austríaco originario de Graz, fue alumno y ayudante de van Swieten en la Universidad de Viena. Varios historiadores afirman que Auembrugger fue el verdadero descubridor de la utilidad de la percusión. Enfrascado en el diagnóstico de las enfermedades del tórax, hacia 1754 ideó la "percusión directa" de la caja torácica con el fin de conocer el estado de los órganos internos mediante sonidos. Era aficionado a la música y estaba acostumbrado a distinguir distintos tipos de tonalidades. Siendo niño ayudaba a su padre, posadero, a golpear barriles de vino pues el sonido que producían les permitía saber si estaban llenos o vacíos. Más tarde siendo ya médico, imaginó que el tórax de sus pacientes podía comportarse como un barril si fuera percutido apropiadamente. A lo largo de siete años de trabajo hospitalario registró las diferencias de tono provocadas por una diversidad de enfermedades de pulmón y corazón, realizando necropsias para corroborar sus hallazgos. Prontamente llevó a cabo experimentos inyectando distintas cantidades de líquido en cadáveres y estudiando los sonidos a que daban lugar en la zona ${ }^{23}$.

Aplicando la metodología de van Swieten, los trabajos de percusión llevaron a Auembrugger a describir un método (Figura 3) para detectar y localizar cavidades tuberculosas en el pulmón. También describió el "frémito", una vibración del tórax producida por la voz o la respiración del enfermo, que puede detectarse colocando la palma de la mano abierta en el pecho del paciente. Su nombre se asocia al Signo de Auenbrugger, una protuberancia de la región epigástrica en casos de derrame pericárdico masivo. Estos descubrimientos 


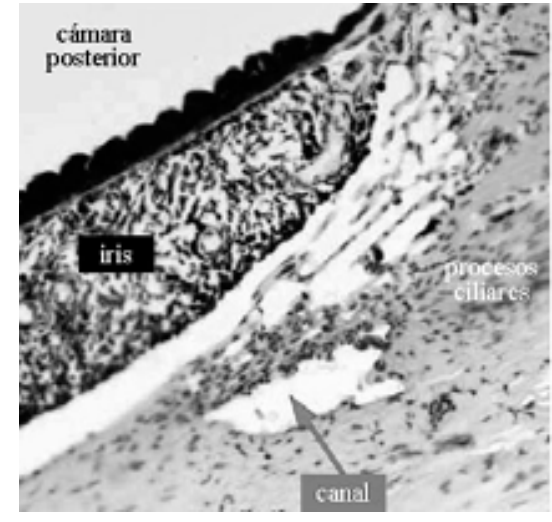

Figura 4. Canal de Schlemm.

fueron publicados en el libro Inventum Novum Ex Percussione Thoracis (1761), aunque la técnica de percusión directa quedó relegada varios años hasta que Corvisart, médico de Napoleón, reconoció su importancia a principios del Siglo XIX ${ }^{23,25}$.

Friedrich Schlemm (1795-1858). Nació en Salzgitter, Alemania, en el seno de una familia pobre, razón por la cual inicialmente no pudo acceder a la educación universitaria. Siendo aprendiz de un barbero-cirujano en Braunschweig, tuvo oportunidad de estudiar anatomía y cirugía en el Instituto Anatomoquirúrgico local. En 1816, aún siendo estudiante y ayudado por un compañero, exhumó el cadáver de una mujer recientemente fallecida y trasladó el cuerpo desde el cementerio hasta la universidad, para estudiar los efectos del raquitismo en los huesos. Por esta acción debió purgar 4 semanas de prisión. El director del Instituto de Anatomía de Berlín, profesor Rudolphi, tomó nota de la destreza de Schlemm para la disección anatómica y lo convirtió en su ayudante, asociación que fructificó en numerosos descubrimientos anatomofisiológicos ${ }^{30}$.

En 1821 obtuvo su título de médico en la Universidad de Berlín, donde se desempeñó como prosector hasta 1833, cuando fue designado profesor de anatomía, cargo que mantuvo durante 25 años, durante los cuales prosiguió sus investigaciones y la formación de futuros médicos. Nunca abandonó sus estudios en cadáveres. Schlemm padecía una ambliopía en su ojo derecho, quizá por eso decidió estudiar la estructura de este órgano. Fue el primero en describir los nervios corneales del ojo y hoy es conocido por el epónimo "Canal de Schlemm" (Figura 4), conducto que recoge el humor acuoso de la cámara anterior y lo vuelca al torrente sanguíneo ${ }^{15,30}$.

Paul Langerhans (1847-1888) fue un berlinés, hijo y hermano de médicos. Comenzó su carrera de medicina en 1865 en la Universidad de Jena, donde fue asistente de Ernst Haeckel (genetista evolucionista, embriólogo y morfólogo). Prosiguió sus estudios en la Universidad de Berlín, contando entre sus profesores a Julius Conheim (descubridor de la migración leuco- citaria en la inflamación) y Rudolf Virchow (patólogo creador de la "teoría celular"), en cuyo laboratorio del Instituto de Patología trabajó como ayudante, estableciendo con él una gran amistad. Las investigaciones de Langerhans se centraron en el estudio de la histología, campo en el que aplicó con éxito las nuevas técnicas de tinción. Investigó la inervación de la piel, describiendo las terminaciones nerviosas del estrato de Malpighi de la epidermis, así como el stratum granulosum, conocido más tarde como Estrato de Langerhans. También se ocupó de los macrófagos, siendo un pionero en el estudio del sistema retículoendotelial. Durante su etapa de estudiante realizó su descubrimiento más conocido, mientras completaba su tesis de graduación (1867). Al investigar en conejos la estructura del páncreas mediante inyecciones de Azul de Prusia distinguió varios tipos celulares relacionados a la secreción de enzimas digestivas. Pero también se percató de la existencia de unas células pequeñas, poligonales, sin gránulos, que tenían el aspecto de manchas diseminadas en el interior del parénquima ${ }^{11}$ (Figura 5).

Langerhans las describió como "células pequeñas, de contenido homogéneo y forma poligonal, núcleo redondeado sin nucléolo y unidas siempre de dos en dos o formando pequeños grupos o montículos, como islotes". En 1889, los alemanes Minkowski y von Mering extirparon el páncreas a un perro, el cual registró la aparición de poliuria y glucosuria ("diabetes"). En 1893, el histólogo francés Laguesse propuso que las células halladas en el páncreas quizá fabricaran algún producto de secreción interna y las denominó "islotes de Langerhans". En 1902 Szobolew y Schultze establecieron que producían un misterioso principio antidiabético, denominándolo "insulina" (de ínsula: isla). Medio siglo después del descubrimiento de Langerhans, los investigadores Banting y Best aislaron por vez primera la hormona secretada en los islotes ${ }^{11,27}$.

Louis-Antoine Ranvier (1835-1922) estudió medicina en Lyon, graduándose en 1865. Poco tiempo después ingresó al Collège de France como discípulo ("preparador") del gran maestro de la fisiología experimental, Claude Bernard, donde trabajó durante casi diez años. A diferencia de su maestro, quien practicaba la vivisección, Ranvier prefirió estudiar los tejidos

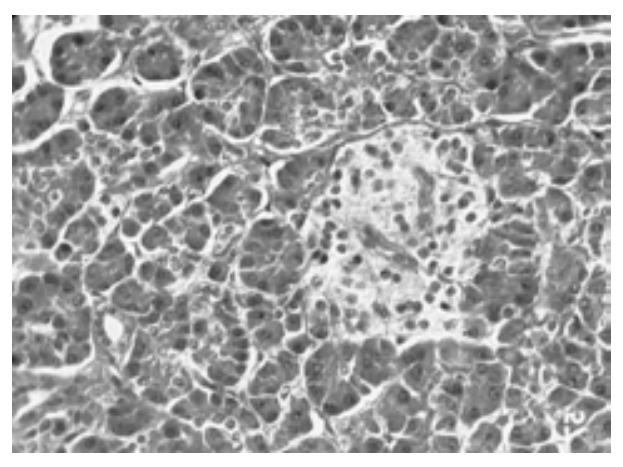

Figura 5. El "islote" de Langerhans. 
a través del microscopio. Investigó temas de anatomía, histología y fisiología. Entre sus descubrimientos más importantes cabe destacar el recubrimiento lipídico de los axones (vaina de mielina) y las estructuras subcelulares que recubren los axones de las neuronas y confieren mayor velocidad al impulso nervioso (nodos de Ranvier), estos últimos visualizados por primera vez en una rana (1878), a partir del nervio ciático tratado con ácido ósmico, los cuales aparecieron como estrangulaciones a intervalos de un milímetro ${ }^{2}$, capaces de conferir mayor velocidad al impulso nervioso (Figura 6).

También describió otras estructuras anatómicas como las células de Merkel-Ranvier (células melanocíticas de la epidermis), la histología de los ganglios linfáticos y la estructura de los tejidos óseo, conjuntivo y muscular. Confirmó la degeneración walleriana de los axones y la neoformación de fibras nerviosas. Sostenía que la regeneración nerviosa era un caso particular de la ley general del crecimiento del centro hacia la periferia. Santiago Ramón y Cajal exaltó la concepción del francés sobre la estructura y fisiología del sistema nervioso. Ranvier describió asimismo la diapédesis de los linfocitos, es decir, su habilidad para atravesar las paredes de los vasos, observando que muchas células se añaden a la linfa cuando ésta pasa a través de un ganglio. Inició la investigación sobre el mecanismo de la contracción muscular; sus hallazgos sobre la estructura del tejido muscular dieron lugar a la obra Leçons d'anatomie générale sur le système musculaire (1880). Con Balbiani, fundó en 1897 la revista científica Archives d'anatomie microscopique. Merced a sus méritos y a propuesta de Bernard, en 1875 ocupó la cátedra de anatomía general del Collège, a los 39 de edad. Ranvier es considerado como uno de los científicos franceses más destacados del siglo XIX; sobresalió por combinar la tradición histológica alemana con la moderna fisiología francesa ${ }^{2}$.

Ivar Viktor Sandström (1852-1889), siendo estudiante de medicina en la Universidad de Uppsala (Estocolmo, Suecia), publicó en 1880 el descubrimiento de las glándulas paratiroides (On a new gland in man and fellow animals'). Hacia 1850, durante una disección de un rinoceronte hindú en el Zoológico de Londres, el biólogo inglés Richard Owen ya había descrito "un pequeño cuerpo compacto de color amarillento y aspecto glandular, adherido a la tiroides en el sitio de emergencia de una vena". Al momento de efectuar su descubrimiento, Sandström no conocía la descripción de Owen, publicada en una revista inglesa de circulación limita-

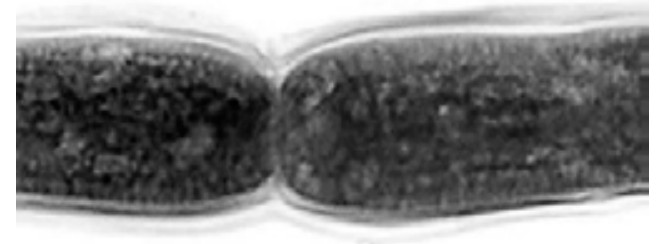

Figura 6. Nodo de Ranvier en nervio periférico.

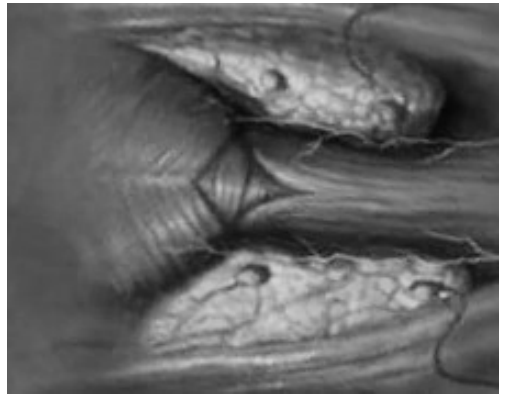

Figura 7. Tiroides y paratiroides.

da. El sueco fue el primero en constatar la existencia de las glándulas paratiroides (Figura 7), inicialmente halladas en perros. Luego, en el lapso 1877-1880, las identificó en el gato, conejo, buey, caballo y finalmente en seres humanos ${ }^{13}$.

Sandström efectuó un detallado estudio anatómico e histológico de las glándulas y, vislumbrando su rol endocrino, expresó "considero probable que se trate de una glándula vascular". A él se le debe la denominación "paratiroides" y la demostración de su estructura glandular. Considerado como el responsable del último descubrimiento anatómico de importancia, Ivar Sandström -quien padecía problemas psiquiátricos- se suicidó en 1889, a la edad de 37 años ${ }^{8}$.

La importancia del descubrimiento de Sandström permaneció ignorada hasta los experimentos de Eugene Gley (1857-1930), quien confirmó la función endocrina de las paratiroides. Fisiólogo y sucesor de BrownSéquard en el Collège de Francia, Gley observó que el tétanos causado por la tiroidectomía experimental en perros sólo ocurría si la extirpación había incluido a las glándulas descritas por Sandström. Tal observación fue la clave para acceder al conocimiento del metabolismo del calcio ${ }^{8,13}$.

Julius Bernstein (1839-1917) egresó como médico en Berlín, en 1862. Había sido el alumno más sobresaliente de Emil Dubois Reymond, por lo cual abrazó la especialidad de la fisiología (electrofisiología) y colaboró en los trabajos de su maestro, el primero en relacionar la conducción nerviosa con la electricidad. En el Instituto de Fisiología de la Universidad de Heidelberg se convirtió en asistente de Hermann von Helmholtz. Juntos descubrieron que la generación de electricidad en los axones de las neuronas no era un producto secundario de su actividad, sino un medio para transmitir mensajes de un extremo a otro. En 1859 lograron establecer la velocidad de propagación de tal transmisión, que resultó ser de $27 \mathrm{~m} / \mathrm{s}^{17}$.

En 1872 Bernstein le sucedió a Friedrich Goltz en el cargo de profesor de fisiología de la Universidad de Halle. Su trabajo se concentró en los campos de la biofísica y neurofisiología, siendo el pionero en el uso de términos como "potenciales de reposo y acción" en nervios y músculos (Figura 8). En 1902 desarrolló la "teoría del potencial de membrana", que fue la primer 


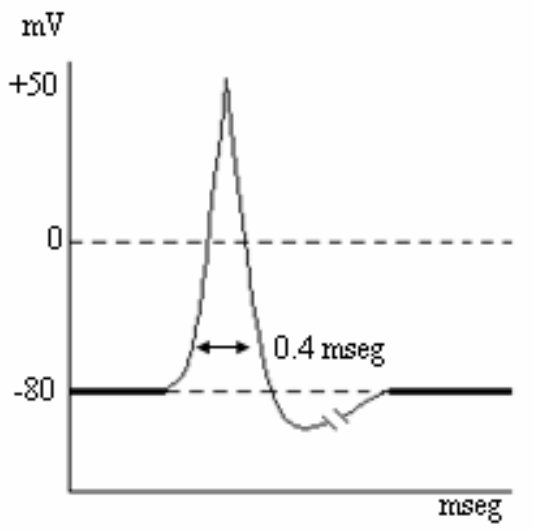

Figura 8. Potencial de acción en el nervio.

explicación fisicoquímica de los fenómenos eléctricos en biología, basada en la permeabilidad selectiva de las membranas celulares hacia distintos iones. Bernstein también inventó un "reótomo diferencial", dispositivo para medir la velocidad de los impulsos bioeléctricos ${ }^{29}$.

Jean-Marie Camille Guérin (1872-1961) nació en Poitiers (Francia) y estudió veterinaria en Alfort, revelando gran interés por las enfermedades infecciosas. Fue alumno y ayudante del infectólogo veterinario Edmond Nocard (1850-1903) quien fue el descubridor del primer actinomiceto aerobio reconocido como patógeno (Nocardia sp.), aislado en 1888 en una epidemia en vacunos. Con él adquirió Guérin profundos conocimientos sobre los microbios y la metodología para estudiarlos. Egresó en 1896 e inmediatamente comenzó a trabajar como discípulo del médico bacteriólogo Léon Charles Albert Calmette, a la sazón director del Instituto de Seroterapia en Lille, merced a una recomendación de Nocard. Guérin participó en la investigación y producción de sueros antivenenosos y de la vacuna antivariólica, mejorando la obtención de esta última al introducir el conejo como huésped intermediario. Asistió a Calmette en el desarrollo de una vacuna contra la tuberculosis. Entre 1905 y 1915 publicó con Calmette un conjunto de trabajos sobre el mecanismo de infección de la tuberculosis. Su objetivo era tornar avirulenta alguna cepa para usarla como vacuna. Los trabajos quedaron interrumpidos a consecuencia de la invasión de la ciudad por los alemanes en 1915, quienes destruyeron o robaron gran parte del material científico del instituto. En 1921 Calmette y Guérin obtuvieron una cepa de bacilos atenuados ("bacilo Calmette-Guérin", $B C G$ ) capaz de conferir inmunidad a la tuberculosis ${ }^{24}$ (Figura 9).

En poco tiempo se habían vacunado exitosamente miles de niños en Francia. En 1933 murió Calmette, cuando en Francia habían sido distribuidas más de 800.000 vacunas y, a partir de 1950 , la vacunación con BCG fue declarada obligatoria en ese país. En 1935 Guérin fue elegido miembro de la Academia de Medicina, ocupando la presidencia en 1951. Durante la segunda guerra mundial, entre 1939 y 1945, vivió en

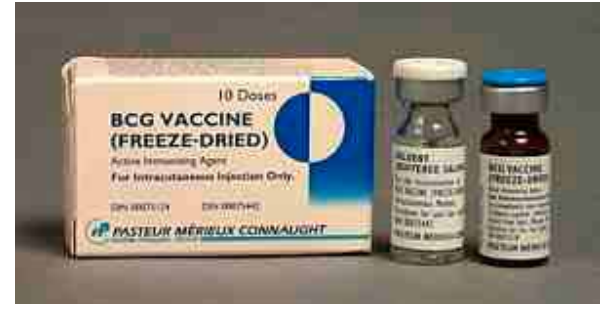

Figura 9. Aparición de la vacuna BCG.

el Instituto Pasteur, después de que su vivienda parisina fuera requisada por el ejército alemán. En 1945 fue miembro del Comité Nacional de Higiene Social (Ministerio de Sanidad Pública) y en 1948 dirigió el primer Congreso Internacional sobre la BCG en París. Fue Presidente de la Academia de Veterinarios de Francia en 1949. La Academia de Ciencias le concedió en 1955 el gran premio a la investigación científica. En 1961 murió en el Hospital Pasteur a los 89 años ${ }^{12,24 .}$

Willard Myron Allen (1904-1993). Nació en Farmington (New York). Estudió química en la Universidad de Hobart y luego medicina en la Universidad de Rochester, en la cual se desempeñó como ayudante alumno de su profesor de anatomía, George W. Corner. Al terminar el primer año de estudios de medicina (1927), Allen fue invitado por Corner a trabajar con él en la identificación de una presunta hormona responsable de los cambios gestacionales. A finales de 1928 Corner y Allen habían procesado cientos de extractos de cuerpo lúteo de conejas y logrado aislar la hormona más adelante denominada "progesterona" (Figura 10), probando que era necesaria para la continuidad de la gestación temprana ${ }^{1,27}$.

El trabajo se publicó en 1929 en el American Journal of Physiology. En el artículo se expresó "los extractos de cuerpo lúteo contienen una hormona que prepara al útero para la recepción de los embriones induciendo la proliferación progestacional del endometrio" y "es evidente que el cuerpo lúteo es un órgano de secreción interna, una de cuyas funciones es provocar la proliferación del endometrio para nutrir o proteger los blastocitos libres y hacer posible su implantación". Más adelante (1932) Allen se graduó de médico con honores. En 1934, cuatro grupos independientes lograron simultáneamente el aislamiento de la progesterona cristalina:<smiles>CC(=O)C1CCC2C3CCC4=CC(=O)CCC4(C)C3CCC12C</smiles>

Figura 10. Estructura de la progesterona. 
Willard Allen y Oskar Wintersteiner (Estados Unidos), Adolf Butenandt y Ulrich Westphal (Alemania), Max Hartmann y Albert Wettstein (Suiza) y Kart Slotta y colaboradores (Alemania). El aislamiento de la hormona requirió grandes cantidades de material; Butenandt usó los cuerpos lúteos de 50.000 cerdas para obtener unos pocos miligramos en estado puro. El nombre de la hormona surgió en la Segunda Conferencia Internacional sobre Estandarización de Hormonas Sexuales en 1935, donde se acordó la denominación "progesterona", que significa "a favor de la gestación". Willard Allen continuó investigando y aportando conocimientos científicos en torno a la histología y fisiología de los órganos reproductores femeninos, obteniendo muchos premios nacionales e internacionales ${ }^{1,27}$.

Dorothy Price (1899-1980) fue ayudante y discípula del profesor Carl R. Moore en la Universidad de Chicago. En 1930 lograron demostrar la relación recíproca entre los testículos y la hipófisis (la testosterona " $T$ " suprimía la secreción de hormona luteinizante "LH”), y luego entre ovarios e hipófisis. Así, quizás por primera vez en la fisiología hormonal quedó planteado el concepto de retroalimentación hormonal (Figura 11), el cual había sido originalmente aplicado a otras disciplinas ${ }^{22}$.

El fenómeno fue conocido como "the Moore-Price negative feedback concept". Después de la muerte de su maestro, Dorothy escribió que lo recordaba vívidamente dado que fue el director de su tesis doctoral en el Departamento de Zoología de la Universidad de Chicago, y luego el científico a quien acompañó muchos años como colaboradora en investigaciones de fisiología de la reproducción. Trabajando en estrecha asociación, Moore y Price imprimieron un elevado nivel a sus investigaciones, lo cual atrajo a numerosos discípulos. Prueba de ello fueron quince estudiantes que lograron su maestría y 33 que completaron su $\mathrm{PhD}^{9}$.

Ewart George Bertram, nacido en Canadá en 1923, fue ayudante alumno de Murray Barr (1908-

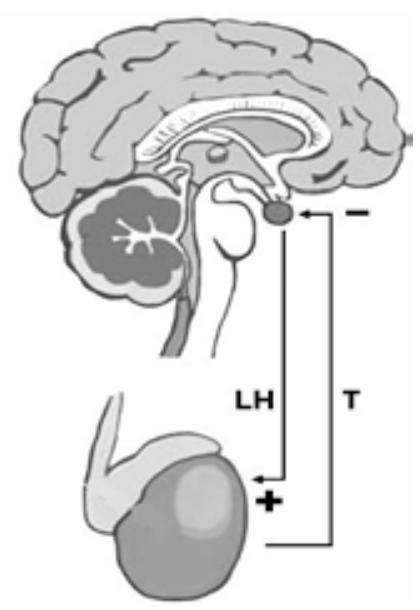

Figura 11. Retroalimentación testículo-hipófisis.
1995), investigador del Departamento de Anatomía de la Universidad de Ontario Occidental. Bertram había servido previamente en las fuerzas armadas durante la segunda guerra mundial. A partir de 1947 colaboró en las investigaciones de Barr, que en ese período realizaba experimentos de neurocitología en gatas, los cuales culminaron con el descubrimiento de la "cromatina sexual". Durante sus trabajos, en 1948 detectaron la presencia de una masa que se teñía intensamente en la periferia del núcleo de las células en interfase. Esta importante estructura celular actualmente se conoce como "Corpúsculo de Barr" (Figura 12). En un principio fue asociado con el nucléolo y llamado "satélite nuclear", término que se utilizó poco tiempo ya que en 1951 Barr reconoció que se observaba asociado al borde interno de la membrana nuclear y que no estaba relacionado con el núcleo ${ }^{3,26}$.

A partir de entonces y debido a su asociación con el sexo femenino se le denominó cromatina sexual o Corpúsculo de Barr, aunque también se conoce como "cromatina X". Los corpúsculos mencionados son masas condensadas de cromatina sexual adheridas al núcleo de las células somáticas de las hembras. De acuerdo a la hipótesis propuesta por Mary Lyon en 1966, uno de los dos cromosomas X en cada célula somática femenina es genéticamente inactivo (sea materno o paterno); los Corpúsculos de Barr representan el cromosoma X inactivo. Los descubridores demostraron que era posible determinar el sexo genético verificando la presencia o ausencia de la masa de cromatina en la superficie interna de la membrana nuclear. Su verificación permite revelar el sexo de un individuo recién nacido con genitales externos no definidos ${ }^{3}$. Ulteriores investigaciones demostraron que el cuerpo de Barr es particularmente distinguible en hembras de primates y carnívoros. Puede estudiarse en tres tipos de células: neuronas, leucocitos polimorfonucleares y células epiteliales (especialmente en la mucosa bucal, donde usualmente es investigado). Otras investigaciones le valieron a Barr varios premios y reconocimientos, incluyendo la nominación para el Premio Nobel de Fisiología y Medicina ${ }^{26}$.

Andrew Fielding Huxley, fisiólogo inglés nacido en 1917, fue discípulo de Alan Lloyd Hodgkin (19141998), biofísico y fisiólogo británico que en ese en-

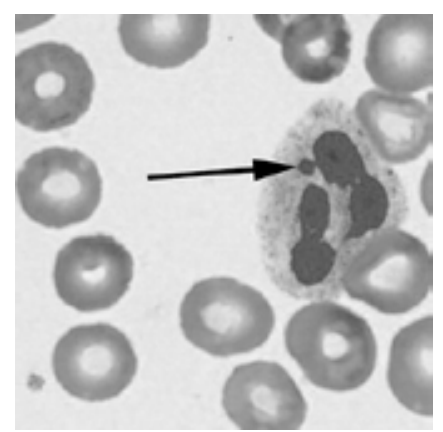

Figura 12. Corpúsculo de Barr (flecha) en un neutrófilo. 


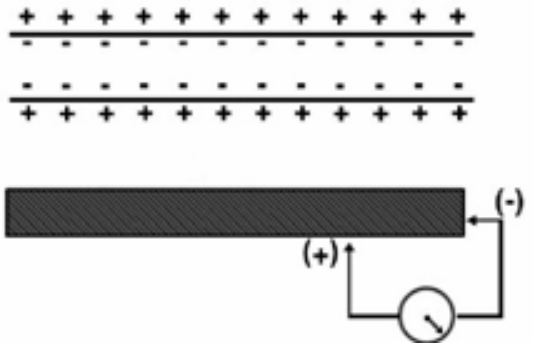

Figura 13. Relación entre iones y bioelectricidad.

tonces investigaba la transmisión nerviosa. En 1938 Hodgkin (investigador) y Huxley (ayudante alumno) integraron un equipo y comenzaron a estudiar la transmisión del potencial de acción de los nervios en el Laboratorio de Biología Marina de Plymouth, Inglaterra. Su primer trabajo fue publicado en Nature, pero a las tres semanas el proyecto fue interrumpido por el inicio de la segunda guerra mundial. Reanudaron sus investigaciones en 1946 y a poco descubrieron un extraordinario modelo experimental: el axón gigante de calamar, nervio de gran diámetro, de hasta $1 \mathrm{~mm}$, dimensión enorme cuando se compara con los 10-20 $\mu \mathrm{m}$ de los axones de mamíferos. Este axón permitía ser insertado con electrodos metálicos para registro e inyección de corriente, se podía exprimir su axoplasma y cambiar la composición del medio intracelular. En 1962 se publicó un trabajo demostrando la generación y propagación del potencial de acción (Figura 13) en un axón al que se le reemplazó el axoplasma por una solución salina con sodio y potasio, en ausencia de ATP ${ }^{14}$.

Los estudios indicaban la existencia de canales iónicos en la membrana celular, los cuales solo fueron aislados décadas después. Además, sentaron las bases para que el fisiólogo suizo Robert Stämpfli evidenciara la conducción saltatoria del impulso nervioso en las fibras mielinizadas. En Cambridge, Huxley continuó estudiando la fisiología de la contracción muscular, postulando la teoría del deslizamiento de los miofilamentos. Desarrolló varios aparatos, entre ellos un microscopio de interferencia para el estudio de las estriaciones de la fibra muscular, un micrótomo para microscopía electrónica y un micromanipulador. En 1963 compartió con Hodgkin el Premio Nobel de Fisiología por sus trabajos sobre los potenciales de acción de los nervios. En 1974 Huxley fue armado caballero por la reina Elizabeth II ${ }^{4,14}$.

Francisco Varela (1946-2001). Nació en Chile, donde estudió biología. Fue alumno y colaborador de Humberto Maturana, médico chileno que propuso que los sistemas vivos son máquinas autopoiéticas, es decir que "están en continua producción de sí mismos, a través de la incesante generación y recambio de sus componentes". Varela efectuó en Harvard su tesis de doctorado, sobre la visión de los insectos. Luego se interesó en los mecanismos neuronales asociados a los fenómenos conscientes, estudiando los procesos de

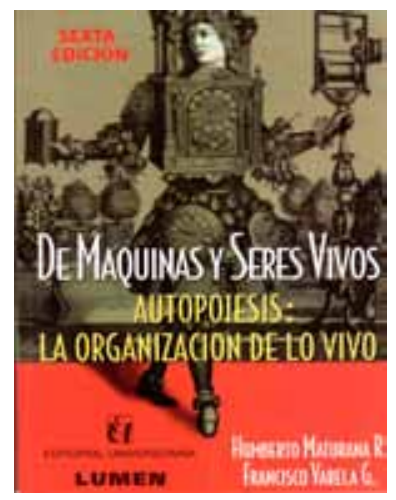

Figura 14. Libro de Maturana y Varela.

sincronía en la actividad nerviosa. Utilizando técnicas matemáticas elaboró un método para predecir ataques de epilepsia, basándose en patrones de electroencefalogramas. En 1970, reunido nuevamente en Chile con su maestro Maturana, estudiaron la génesis de los fenómenos cognitivos. Investigaron los sistemas biológicoperceptivos de distintos animales, encarando las bases del proceso de aprendizaje humano desde una perspectiva biológica ${ }^{5}$. En conjunto publicaron varios libros, entre ellos De máquinas y seres vivos (1972) (Figura 14) y El árbol del conocimiento (1984).

Competente matemático, Varela formalizó el concepto de autonomía de los sistemas biológicos (especialmente inmunitario y nervioso). Postuló que las experiencias concientes constituyen una propiedad emergente propia de un grupo transitorio de neuronas ("resonantes") que aparecen y desaparecen sin cesar. Para la escuela epistemológica del determinismo estructural, el concepto de autopoiesis creado por Maturana y Varela describe una condición de autogeneración de los seres vivos relacionada con los cambios estructurales. "Los seres vivos son verdaderos remolinos de producción de componentes, por lo que las sustancias que se toman del medio o se vierten en él, pasan participando transitoriamente en el ininterrumpido recambio de componentes". "Los seres vivos son sistemas autopoiéticos, y están vivos sólo mientras están en autopoiesis" ". El término autopoieis ("que se hace a sí mismo") fue concebido para definir la continuidad de la materia viva. Así como los ecosistemas están comprendidos en redes de alimentación, en fisiología los organismos están inmersos en redes de aparatos, órganos y células. Y las células en redes de moléculas, sustentándose a sí mismas por redes de reacciones químicas que producen la totalidad de los componentes celulares. Más de 200 publicaciones y 15 libros reflejan la dilatada obra de Varela. Durante sus últimos años se abocó a la búsqueda de los fundamentos biológicos de la conciencia, un tema antes casi vedado en la investigación científica. Varela murió en París a los 54 años ${ }^{5,20}$.

Charles Herbert Best (1899-1978), estudiante de medicina nacido en Maine (EEUU), a los 22 años de edad se incorporó como asistente de un joven médi- 


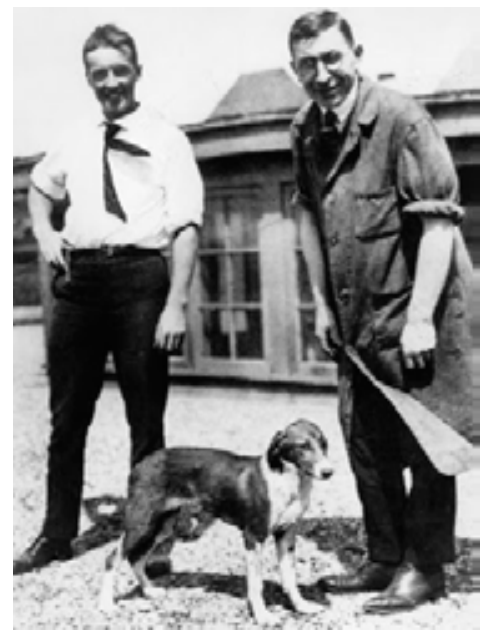

Figura 15. Best, Banting y la perrita Marjorie.

co canadiense de 30 años llamado Frederick Banting (1891-1941) en el Instituto Fisiológico de la Universidad de Toronto, Canadá. Hijo de padres canadienses, Best estudiaba en dicha universidad cuando surgió la posibilidad de realizar una pasantía con el Dr. Banting, quien estaba investigando el funcionamiento del páncreas en búsqueda del misterioso "principio antidiabético" mencionado por Szobolew y Schultze en $1902{ }^{21}$. En esencia, el proyecto consistía en obtener un extracto de tejido pancreático de bovinos y con él controlar la diabetes en caninos, para luego intentar la curación de diabéticos humanos. John McLeod, director del instituto, cedió los laboratorios y colaboró en la investigación. Para la purificación de los extractos de páncreas intervino el bioquímico James Collip. El 27 de julio de 1921, una perrita diabética llamada Marjorie, en coma y casi moribunda, recibió el extracto pancreático y al poco tiempo mejoró (Figura 15). Varias horas después de la inyección, la glucosuria reveló una drástica reducción. Como un reguero de pólvora, la noticia se difundió por el mundo entero. El principio activo, llamado "isletina" por Banting, fue ulteriormente experimentado con éxito en seres humanos ${ }^{27}$.

En 1923 se otorgó el Premio Nobel de Fisiología y Medicina a Banting y McLeod, no así a Best por no haber concluido aún su carrera universitaria (egresó como médico recién en el año 1925). Banting compartió con Best la mitad del dinero que le correspondía por el premio y McLeod hizo lo propio con Collip. Tiempo después Banting se dedicó a investigar la fisiología pulmonar y a los 49 años murió en un accidente aéreo mientras se desempeñaba como combatiente en la segunda guerra mundial. McLeod se abocó al metabolismo de los carbohidratos y escribió once libros, entre ellos $R e$ cent Advances in Physiology. Por su parte, Best efectuó un postdoctorado en Inglaterra y, cuando McLeod se retiró de la Cátedra de Fisiología de Toronto (1929), fue designado en dicho puesto, llegando a ser director del Departamento de Investigación Médica en 1941. Sus líneas de investigación posteriores se enfocaron en la heparina y el metabolismo de los carbohidratos. Fue el descubridor de la colina y de la enzima histaminasa ${ }^{21}$, 27 . En 1937 Charles Best y Norman Taylor publicaron The Physiological Basis of Medical Practice, libro que (con otros editores) aún continúa siendo una de las más importantes bases de datos para el estudiante de ciencias de la salud (la $14^{\circ}$ edición se publicó en 2010).

\section{COLOFÓN}

Obviamente no es irrefutable que la formación de recursos humanos sea una prerrogativa del Siglo XXI (ni siquiera de los siglos XIX y XX), desde el momento que existió a partir de los albores de la ciencia. Quizás lo novedoso sea que tal actividad se haya transformado en un requisito trascendente al momento de evaluar los antecedentes de investigadores y profesores universitarios (cantidad de auxiliares, ayudantes, adscriptos, pasantes, becarios y tesistas dirigidos). Infortunadamente, en Argentina un director se valora por el número de discípulos admitidos (cantidad) pero no existen parámetros para deducir quizás lo más importante, los resultados de la tarea de formación (calidad). Otro contrasentido reside en que, desde bastante tiempo atrás, la tan mentada e imperativa formación de recursos humanos se torna abstracta e infructuosa cuando el postulante, luego de años de arduo esfuerzo, tropieza con las restricciones derivadas de una abarrotada planta universitaria o con la exigüidad de ofertas por parte de los organismos de investigación científica.

Una ímproba tarea en la formación de recursos humanos es la admisión o rechazo del eventual postulante, dado el "encandilamiento" que ocasiona el buen desempeño académico ("curricular") en el mejor de los casos, o -en el peor- la presunción de carencia de cualidades y aptitudes que lo califiquen para la investigación científica. La selección es difícil y puede conducir a errores hasta en científicos con las mentes más preclaras y capacitadas para esta labor. En una conferencia dictada en 1984, Luis Federico Leloir (Premio Nobel de Química 1970) rememoró que en el año 1956 había rechazado una solicitud para dirigir la tesis doctoral de un licenciado en química recientemente egresado de la Universidad de Buenos Aires. Admitió haber cometido "uno de mis grandes errores" porque lo evaluó como "un estudiante no especialmente bueno, que se preocupaba más por la política y el centro de estudiantes...". El joven era César Milstein, quien en 1984 obtuvo el Premio Nobel de Fisiología y Medicina por sus trabajos de inmunología en el área de los anticuerpos monoclonales ${ }^{18}$.

\section{REFERENCIAS}

1. Allen WM, Corner GW. 1929. Normal growth and implantation of embryos after very early ablation of the ovaries, under the influence of extracts of the corpus luteum. Am J Physiol 88: 340-346. 
2. Appel TA. 1975. Louis-Antoine Ranvier, In: Dictionary of scientific biography (Gillispie C ed), Scribner's, New York, p. 295-297.

3. Barr ML, Bertram EG. 1949. A morphological distinction between neurones of the male and female. Nature 163 : 676-677

4. Cole KS. 1968. Membranes ions and impulses, Univ Calif Press, Berkeley, p. 114.

5. Coppo JA. 2005. Fundamentos y metodología de la investigación científica, tomos 1 y 2, Moglia Ediciones, Corrientes (Arg.), $862 \mathrm{p}$.

6. Coppo JA. 2012. El rumbo epistémico de la fisiología, EUCASA, Salta (Arg.), 377 p.

7. Diepgen P. 1932. Historia de la medicina, $2^{\mathrm{a}}$ ed., Labor, Barcelona, $608 \mathrm{p}$.

8. Eknoyan G. 1995. A history of parathyroid glands. Am J Kidney Dis 26: 801-807.

9. Freeman E, Bloom D, McGuire E. 2001. A brief history of testosterone. J Urol 165: 371-373.

10. Günther E. 1978. The historical success of Johan Ham. Dermatol Monatsschr 164: 585-589.

11. Gutiérrez GA. 2002. Del macroscopio al microscopio: historia de la medicina cientifica, Ed Javegraf, Bogotá, p. 435.

12. Hawgood BJ. 1999. Doctor Albert Calmette 1863-1933: founder of antivenomous serotherapy and of antituberculous BCG vaccination. Toxicon 37: 1241-1258.

13. Johansson H. 2009. Parathyroid history and the Uppsala anatomist Ivar Sandström. Med Secoli 21: 387-401.

14. Kotsias BA. 2004. El axón gigante de calamar. Medicina (Buenos Aires) 64: 3.

15. Kretschmann HJ. 1958. Friedrich Schlemm on the 100th anniversary of his death. Anat Anz 105: 282-291.

16. Kuhn TS. 1985. La estructura de las revoluciones científicas, FCE, México, 307 p.
17. Lazorthes G. 1981. Le système nerveux périphérique, Masson, Paris, p. 307-318.

18. Leloir LF. 1984. Conferencia en la Facultad de Medicina de la UBA. Acta Bioq Clin Lat 18: 553-554.

19. Lindeboom GA. 1973. Los grandes sistemáticos: Hermann Boerhaave. En: Historia Universal de la Medicina (Laín P. ed), Salvat, Barcelona, p. 319-325.

20. Luhmann NR. 1997. Organización y decisión, autopoiesis y entendimiento comunicativo, Anthropos, Barcelona, p. 173-174.

21. Lyons AS, Petrucelli RJ. 1994. Historia de la medicina, Mosby, Barcelona, $615 \mathrm{p}$.

22. Moore CR, Price D. 1938. Some effects of testosterone and testosterone-propionate in the rat. The Anat Rec 71: 59-78.

23. Multanosky MP. 1967. Historia de la medicina, Ed Acad Cient Cuba, La Habana, p. 288-290.

24. Pérez OA. 2004. Historia de la veterinaria, Ed. Allignani, Santa Fe (Arg), 485 p.

25. Pontificia Universidad Católica de Chile. 1996. Apuntes de Historia de la Medicina. On line: http://escuela.med. puc.cl/publ/HistoriaMedicina/Default.html.

26. Potter P, Soltan H. 1997. Murray Llewellyn Barr. Biograph Mem Fellows Royal Soc 43: 33.

27. Roca AJ. 2008. Historia de las hormonas, Ed. Acad Nac Med Colombia, Bogotá, 199 p.

28. Rostand J. 1985. Introducción a la historia de la biología, Planeta, Barcelona, 352 p.

29. Seyfarth EA. 2006. Julius Bernstein (1839-1917): pioneer neurobiologist and biophysicist. Biol Cybern 94: 2-8.

30. Winkelmann A. 2008. Schlemm, el ladrón de cadáveres. Ann Anat 190: 223-229. 